\title{
Less Strain Energy despite Fewer Misfit Dislocations: The Impact of Ordering
}

\author{
Th. Schmidt, ${ }^{*}$ R. Kröger, J. I. Flege, T. Clausen, and J. Falta \\ Institute of Solid State Physics, University of Bremen, Otto-Hahn-Allee 1, 28359 Bremen, Germany \\ A. Janzen, P. Zahl, ${ }^{\dagger}$ P. Kury, M. Kammler, and M. Horn-von Hoegen \\ Institute of Laser and Plasma Physics, University of Duisburg-Essen, Universitätsstrasse 5, 45117 Essen, Germany
}

(Received 10 August 2005; published 13 February 2006)

\begin{abstract}
The average strain state of Ge films grown on $\mathrm{Si}(111)$ by surfactant mediated epitaxy has been compared to the ordering of the interfacial misfit dislocation network. Surprisingly, a smaller degree of average lattice relaxation was found in films grown at higher temperature. On the other hand, these films exhibit a better ordered dislocation network. This effect energetically compensates the higher strain at higher growth temperature, leading to the conclusion that, apart from the formation of misfit dislocations, their ordering represents an important channel for lattice-strain energy relaxation.
\end{abstract}

PACS numbers: 68.55.-a, 61.10.-i, 68.37.Lp

For the accommodation of two lattices with different lattice constants, the introduction of interfacial misfit dislocations (MDs) is a well established mechanism. As soon as a critical layer thickness $t_{c}$ is exceeded during growth, the energy necessary for dislocation formation is overbalanced by the elastic energy gained by such a plastic relaxation. Hence, at $t_{c}$ a transition from pseudomorphic (coherent) to relaxed (incoherent) growth is predicted. If the formation and movement of MDs is not suppressed by kinetic limitations, a regular arrangement of MDs can be expected. This has been observed in a variety of heteroepitaxial systems, such as $\operatorname{Ag}(111) / \mathrm{Si}(001)$ [1], $\mathrm{CoSi}_{2} / \mathrm{Si}(111)$ [2], $\mathrm{Ag} / \mathrm{Pt}(111)$ [3], and $\mathrm{Fe} / \mathrm{W}(110)$ [4,5]. The strain fields of regularly arranged MDs, the critical layer thickness, and their dependence on the lattice mismatch have been investigated in great detail [6-8]. However, less attention has been paid to possible effects of disordered MDs. In the present Letter, we will show that deviations from perfect periodic order of the MD network can lead to a significant increase of the strain energy.

As an example system, $\mathrm{Ge} / \mathrm{Si}(111)$ was studied. Apart from its great importance for technological applications [9], this is an ideal model system for the epitaxy of lattice mismatched materials, for three reasons: First, the lattice mismatch of $4.2 \%$ is moderate in comparison, e.g., to InAs/GaAs or $\mathrm{CdSe} / \mathrm{ZnSe}$ (both about $7 \%$ ) or $\mathrm{InN} / \mathrm{GaN}$ $(11 \%)$. Second, the bonds of the elemental semiconductors have purely covalent character, unlike those of the compounds, so that neither differences in ionicity nor straininduced electric fields have to be taken into account [10]. Third, in the case of surfactant mediated epitaxy (SME) [11], the intermixing of $\mathrm{Si}$ and $\mathrm{Ge}$ can be neglected, allowing for atomically sharp interfaces and $\delta$ layers $[12,13]$. The $\mathrm{Ge} / \mathrm{Si}(111)$ interface is of special interest, since in this orientation the MDs can glide in the interfacial plane and can, therefore, form a periodic network [14]. For SME growth of Ge on the Si(111) surface, the formation of such periodic interfacial dislocation networks has already been observed by different experimental techniques [1421].

The Ge films investigated here were grown by SME under ultrahigh vacuum conditions, with $\mathrm{Sb}$ codeposited as a surfactant. The growth temperature $T_{\mathrm{G}}$, as determined by infrared pyrometry, was varied from 490 to $720^{\circ} \mathrm{C}$. The Ge film thicknesses, determined by in situ electron diffraction [22], were found to be around $100 \AA$.

To characterize the strain state of the Ge films and to investigate the properties of the misfit dislocation network, grazing incidence $\mathrm{x}$-ray diffraction (GIXRD) and transmission electron microscopy (TEM) were employed. More details about the sample preparation and the experimental setup have been published previously [20,21].

Reciprocal space maps (RSMs) in the $q_{\|}$plane, recorded by GIXRD in the vicinity of the (22/4) Bragg reflection, are shown in Fig. 1 for different growth temperatures. Because of the finite scattering vector component perpendicular to the surface, the diffraction spots in the RSMs do not represent Bragg spots but the intersections of crystal truncation rods (CTRs) with the $q_{\|}$plane. In each of the RSMs, the Si (30) CTR appears as a sharp feature at $q_{\|}=$ 3.0 r.l.u.; the rather elongated shape is due to the instrumental response function. For low growth temperatures $T_{\mathrm{G}}$, the Ge spot at $q_{\|} \approx 2.9$ r.l.u. is weak and very broad. With increasing $T_{\mathrm{G}}$, the Ge spot becomes stronger and narrower. Further, a hexagonal array of satellite spots evolves, which is clearly visible at $T_{\mathrm{G}}=590^{\circ} \mathrm{C}$. The presence of these satellites is intimately related to a periodic network of misfit dislocations [17,20-22]. Accordingly, the evolution of the satellite spots reflects a transition from rather disordered MDs to an ordered MD network. On careful inspection, satellite spots can already be recognized for $T_{\mathrm{G}}=540^{\circ} \mathrm{C}$.

The widths of the satellite spots and the Ge spot are indicative of the disorder of the MD network. Figure 2(a) shows the width of the Ge (30) CTR as a function of the growth temperature $T_{\mathrm{G}}$. A decrease of the reflection width 

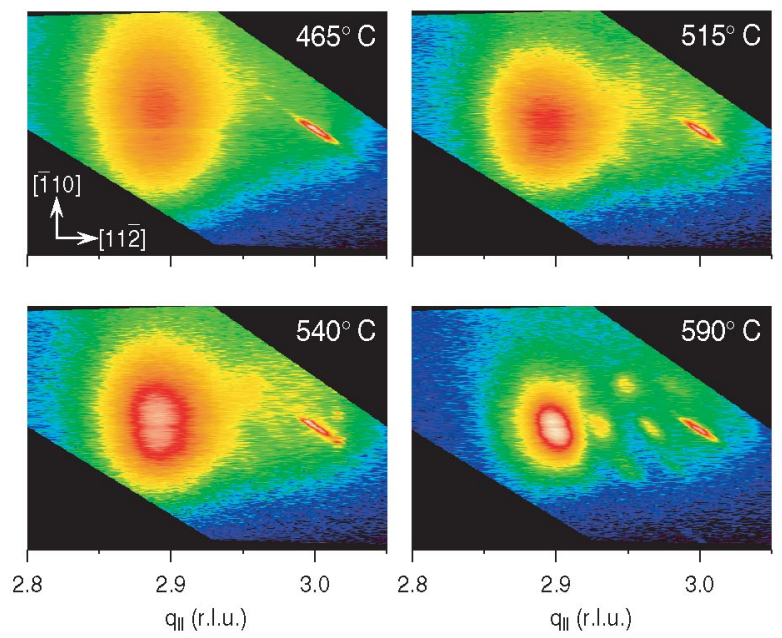

FIG. 1 (color). Reciprocal space maps in the $q_{\|}$plane in the vicinity of the $(22 \overline{4})$ reflection [i.e., near the (30)-CTR, at $q_{\perp} \approx$ $0.05 \times 2 \pi / 3.136 \AA$ ] for Ge films grown on $\mathrm{Si}(111)$ by Sb-SME at different temperatures $T_{\mathrm{G}}$. Scale given in reciprocal lattice units, 1 r.l.u. $=2 \pi / 3.326 \AA$.

is found with increasing $T_{\mathrm{G}}$. This result is explained by an increase in the thermally activated mobility of the dislocations at higher $T_{\mathrm{G}}$, leading to an enhanced ordering. The same arguments should also hold for the formation of dislocations. At higher $T_{\mathrm{G}}$, kinetic limitations of this formation process should become less important, leading to a higher density of MDs and, hence, a diminished average strain of the Ge film. Experimentally, the opposite behavior is observed. From the position of the Ge (30) spot, one can directly derive the average lateral Ge lattice parameter $a_{\mathrm{Ge}}$ and, hence, the strain parameter

$$
\gamma=\frac{a_{\mathrm{Ge}}-a_{\mathrm{Ge}}^{0}}{a_{\mathrm{Si}}^{0}-a_{\mathrm{Ge}}^{0}},
$$

where $a_{\mathrm{Ge}}^{0}$ and $a_{\mathrm{Si}}^{0}$ are the corresponding bulk values for $\mathrm{Ge}$ and $\mathrm{Si}$, respectively. (Hence, $\gamma=0$ for a fully relaxed film and $\gamma=1$ for a fully strained film.) The results are shown in Fig. 2(b). Here data are depicted for an extended set of

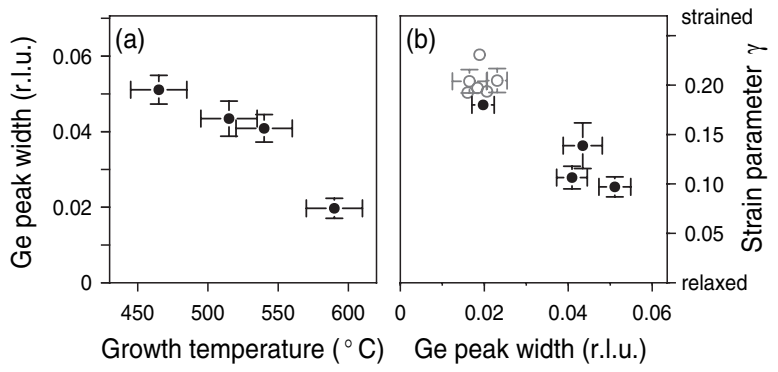

FIG. 2. (a) Dependence of the width (FWHM along the [112] direction) of the Ge (30) CTR on $T_{\mathrm{G}}$, as determined from the reciprocal space map data shown in Fig. 1. (b) Remaining strain $\gamma$ determined by GIXRD versus peak width. Open symbols represent data obtained from other samples. samples, with slightly different preparation, such as growth with $\mathrm{Bi}$ instead of $\mathrm{Sb}$ as surfactant, change of $T_{\mathrm{G}}$ during $\mathrm{Ge}$ deposition, or growth on vicinal $\mathrm{Si}(111)$ substrates. In order to eliminate $T_{\mathrm{G}}$ from the evaluation, Fig. 2(b) shows the strain parameter as a function of the Ge peak width rather than of the growth temperature. From this graph, it is evident that better ordered Ge films exhibit larger remaining strain.

These findings were also confirmed by TEM. Figure 3 shows plan-view TEM images for different growth temperatures $T_{\mathrm{G}}$. The dotted contrast arises from the moiré pattern generated by the superposition of the $\mathrm{Ge}$ and the Si lattice. Obviously, this pattern becomes more regular with increasing $T_{\mathrm{G}}$. This is further supported by the respective autocorrelations of the TEM images. For higher $T_{\mathrm{G}}$, more correlation peaks of higher order are visible than for lower $T_{\mathrm{G}}$.

The average MD separation $L$ can be estimated from high-resolution cross-sectional TEM images (not shown here). The results are represented in Fig. 4. With increasing $T_{\mathrm{G}}$, an increasing separation (i.e., a decreasing density) of MDs is observed. In agreement with GIXRD, this implies a higher lateral strain for higher growth temperatures and, hence, for better ordered films.

Since the misfit dislocations are formed during growth, the lattice mismatch at the growth temperature has to be considered. According to reference data [23], the mismatch increases from $4.35 \%$ at $450{ }^{\circ} \mathrm{C}$ to $4.41 \%$ at $650{ }^{\circ} \mathrm{C}$. Therefore, the driving force for MD formation increases accordingly with increasing temperature. We would, therefore, expect the MD density to increase with temperature, whereas our experimental results show just the opposite effect. Additional relaxation mechanisms might serve as an explanation. For example, intermixing of $\mathrm{Si}$ and $\mathrm{Ge}$ would decrease the strain, and intermixing should become more important for higher growth temperatures, in agreement with our results. For SME, however, $\mathrm{Si} / \mathrm{Ge}$ interdiffusion can be ruled out $[13,24,25]$. An alternative relaxation mechanism could be the formation of additional defects, such as twins and stacking faults. However, as we have shown recently [21], the density of such defects is drastically reduced at higher growth temperatures.
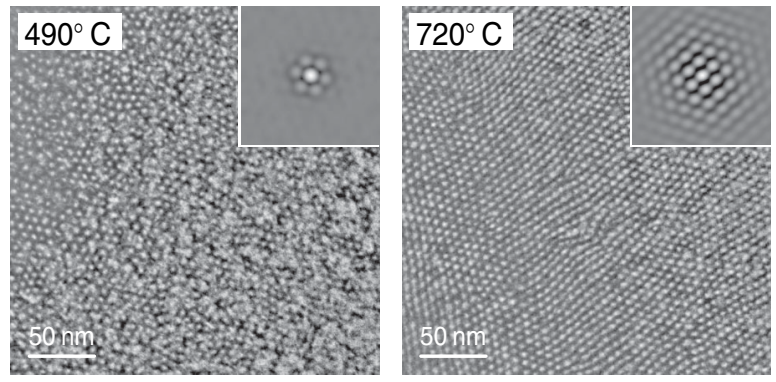

FIG. 3. Plan-view TEM images from Ge films grown by SbSME at different temperatures. For each image, the autocorrelation is shown at larger scale in the inset. 


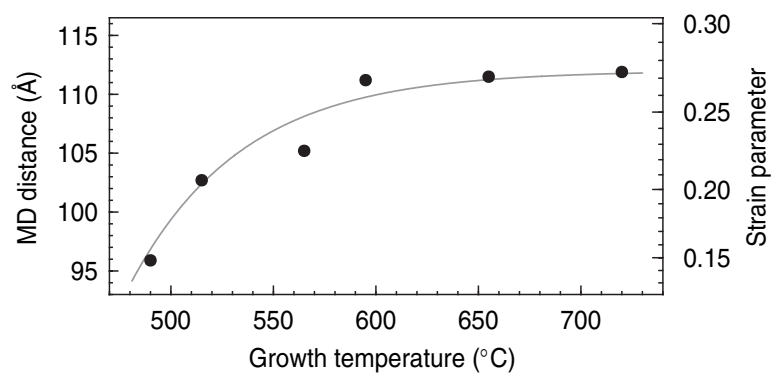

FIG. 4. Average distance $L$ between MDs, as determined from high-resolution cross-sectional TEM, as a function of the growth temperature. The solid line is to guide the eye. The average strain parameter $\gamma$ was calculated according to $f(1-\gamma)=b /(L-b)$, where $f=\left(a_{\mathrm{Ge}}^{0} / a_{\mathrm{Si}}^{0}\right)-1$ is the misfit and $b=3.326 \AA$ is the $\mathrm{Si}$ atomic row distance.

To explain the experimental results, the decisive factor is not the mean strain but the strain energy. In a thermodynamic approach, it is the energy (neglecting entropy effects) which is to be minimized. The strain energy increases quadratically with increasing strain. With respect to the average strain energy $\langle w\rangle$ (per unit area), this implies

$$
\langle w\rangle \propto\left\langle\varepsilon^{2}\right\rangle=\langle\varepsilon\rangle^{2}+\sigma_{\varepsilon}^{2} .
$$

Hence, not only the average strain $\langle\varepsilon\rangle$ but also the standard deviation $\sigma_{\varepsilon}=\sqrt{\left\langle(\varepsilon-\langle\varepsilon\rangle)^{2}\right\rangle}$ of the strain have to be taken into account in calculating the strain energy. Thus, any deviation from a perfectly regular arrangement of MDs will increase the strain energy. In terms of thermodynamics, the system will then try to approach equilibrium, which can be accomplished (under the boundary condition of a disturbed ordering of the MDs) by the formation of more dislocations. From a more microscopic point of view, the probability for the nucleation of additional MDs is governed by the local strain energy.

Equation (2) describes the fundamental principle valid for any heteroepitaxial system. Aiming at a more quantitative description for $\mathrm{Ge} / \mathrm{Si}(111)$, we performed atomistic model calculations. For a perfectly ordered MD network, the system can be divided into identical slabs or supercells, separated from each other by dislocation lines. In the present case, for each of these supercells, up to $10^{6}$ atoms have to be considered. Hence, first principle approaches cannot be applied. Instead, the formalism presented by Keating [26] was employed. Slabs of $N_{1} \times N_{2}=(10 \times$ $10)$ up to $(100 \times 100)$ surface unit cells were considered, with $60 \mathrm{ML} \mathrm{Ge}(\approx 100 \AA)$ and $40 \mathrm{ML}$ Si substrate thickness. The lowest Si bilayer atoms were kept fixed at bulk positions, whereas all other atoms were allowed to relax in all directions. Thicker substrate layers did not yield any significant change in the results. Keating's formalism does not take into account energy contributions from interfacial dangling bonds or due to any reconstruction of dislocation cores. Hence, in the results presented here, such electronic parts of the dislocation line energy are neglected. Both nondissociated dislocations and Shockley partial dislocations have been modeled. The results for the latter configuration are presented below. (Similar results, but with a higher total energy, were obtained for the former dislocation type, emphasizing the general implications of the ansatz.) In the (111) plane, periodic boundary conditions were applied in order to calculate the energies $W\left(L_{1}, L_{2}\right)$ for a slab of lateral size $L_{1} \times L_{2}$. These boundary conditions correspond to a perfectly ordered MD network.

In a second step, the energies so obtained were used to calculate the mean energy per unit area $\langle w\rangle$ according to

$$
\langle w\rangle=\frac{\int_{0}^{\infty} d L_{1} \int_{0}^{\infty} d L_{2} p\left(L_{1}\right) p\left(L_{2}\right) W\left(L_{1}, L_{2}\right)}{\int_{0}^{\infty} d L_{1} \int_{0}^{\infty} d L_{2} p\left(L_{1}\right) p\left(L_{2}\right) \alpha L_{1} L_{2}},
$$

where $p\left(L_{i}\right)$ is the distribution function of the slab length $L_{i}$ in the direction $i$, and $\alpha L_{1} L_{2}$ is the area of the slab (i.e., here $\alpha=\sqrt{3} / 2$ ). Equation (3) is based on the assumption that the strain (equivalently: the lattice parameter) in one supercell does not depend on the size of adjacent supercells. This assumption is supported by the observed shortrange order of the MD network [18], which implies that the (lattice-strain mediated) interaction between MDs is dominated by nearest-neighbor contributions.

In order to evaluate Eq. (3), a distribution function $p(L)$ has to be chosen. Here we used the Gamma distribution (see, e.g., Ref. [27]), which is depicted in the inset in Fig. 5 for different values of its relative standard deviation $\sigma_{L} /\langle L\rangle$.

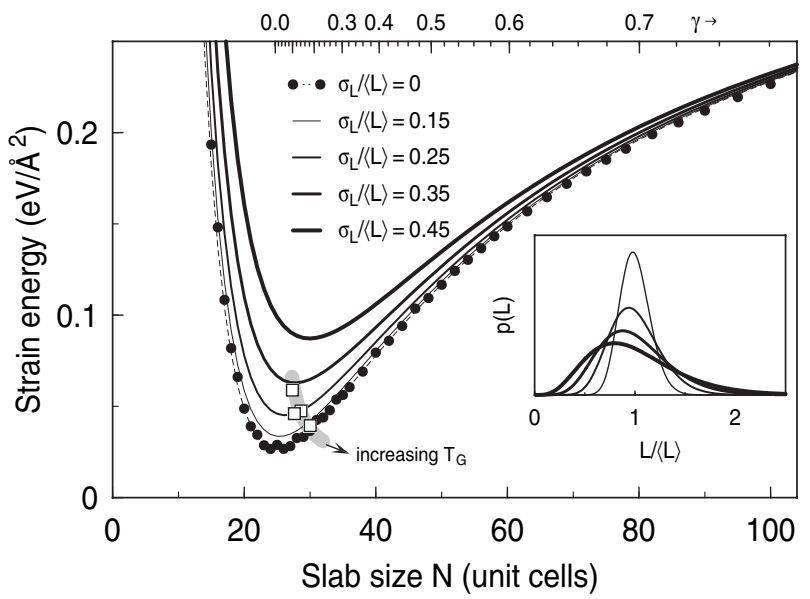

FIG. 5. Strain energy (per area) for slabs consisting of 40 atomic layers of $\mathrm{Si}$ ( $N^{2}$ atoms per layer) and 60 layers $\mathrm{Ge}$ $\left[(N-1)^{2}\right.$ atoms per layer]. MD distances $L$ are proportional to $N$. Solid circles: Minimum energy obtained by atomistic calculations for a single slab (ordered network); the dashed line is a fit. Solid lines: Energy for disordered networks as a function of the average size $N$. Corresponding MD distance distribution functions are shown in the inset. Open square symbols represent the strain energy of samples grown at different temperatures $T_{\mathrm{G}}$ (cf. Fig. 1). 
The dependence of the strain energy on $\sigma_{L} /\langle L\rangle$ is illustrated in Fig. 5. Even for a perfectly ordered network (see solid circles), the strain energy does not drop to zero for the optimal MD distance. Though the average strain is zero for this condition, the covalent interfacial bonds lead to a modulation of the lattice parameters, i.e., to a strain modulation within each slab. According to Eq. (2), this already leads to a finite strain energy. If an additional variation of the strain between different slabs is taken into account, significant increases of the energy are obtained. Irrespective of the MD ordering, the strain energy tends towards the pseudomorphic value for large MD distances, whereas the energy at the optimum distances increases drastically with the disorder. Hence, especially for almost relaxed films such as the ones presented above, an increase in the strain energy arising from the average strain can easily be compensated by a slightly enhanced ordering of the MD network, i.e., by dislocation motion. If, however, such movement of MDs is impeded, e.g., by pinning of MDs at other defects found at a lower temperature [21], more MDs have to be introduced in order to reduce the strain energy. Especially for these kinetically limited conditions, the local properties become important. For example, in a film region with a large distance between two pinned MDs, it is energetically favorable to introduce another MD between them, even if this might lead to a slight local over-relaxation $\left(\gamma_{\text {local }}<0\right)$ which is stabilized by the pinned surrounding MDs. Such local effects might explain why the average strain (not to be confused with the average strain energy) can be smaller at lower than at higher growth temperatures.

Based on the relaxed slab atomic configurations, XRD simulations were performed using the Born approximation. From a comparison of these results (not shown) with the measured Ge (30) peak widths [cf. Fig. 2(b)], the relative standard deviations of the MD distance distribution have been extracted. Since the average strain is also known from the diffraction experiments, the strain energy can be calculated according to the model explained above. The result is also shown in Fig. 5 (see open squares), confirming that $\mathrm{Ge}$ films grown at higher temperatures indeed have less strain energy despite fewer misfit dislocations.

Summarizing, we have shown that the average strain of heteroepitaxial layers alone is not sufficient to describe their degree of relaxation and that not only the formation but also the ordering of misfit dislocations can be regarded as an elastic energy relaxation mechanism.

Part of this work was supported by the Deutsche Forschungsgemeinschaft (Grants No. Fa 363/6 and No. Ho 1611/10). We thank Professor P. L. Ryder for careful reading of the manuscript.
*Electronic mail: tschmidt@physik.uni-bremen.de

${ }^{\dagger}$ Present address: Center for Functional Nanomaterials, Brookhaven National Laboratory, Upton, NY 119735000, USA.

[1] M. Horn-von Hoegen, Th. Schmidt, G. Meyer, D. Winau, and K.H. Rieder, Phys. Rev. B 52, 10764 (1995).

[2] R. Stalder, H. Sirringhaus, N. Onda, and H. von Känel, Appl. Phys. Lett. 59, 1960 (1991).

[3] H. Brune, H. Röder, C. Boragno, and K. Kern, Phys. Rev. B 49, R2997 (1994).

[4] U. Gradmann and G. Waller, Surf. Sci. 116, 539 (1982).

[5] R. Popescu et al., Phys. Rev. B 68, 155421 (2003).

[6] J.H. van der Merwe, J. Appl. Phys. 34, 117 (1963); 34, 123 (1963).

[7] J. W. Matthews, J. Vac. Sci. Technol. 12, 126 (1975).

[8] R. People and J. C. Bean, Appl. Phys. Lett. 47, 322 (1985); 49, 229 (1986).

[9] D. Reinking, M. Kammler, M. Horn-von Hoegen, and K. R. Hofmann, Jpn. J. Appl. Phys. 36, L1082 (1997).

[10] R. M. Martin, Phys. Rev. B 1, 4005 (1970).

[11] M. Copel, M. C. Reuter, E. Kaxiras, and R. M. Tromp, Phys. Rev. Lett. 63, 632 (1989).

[12] J. Falta, T. Gog, G. Materlik, B. H. Müller, and M. Hornvon Hoegen, Phys. Rev. B 51, 7598 (1995).

[13] J. Falta et al., Appl. Phys. Lett. 68, 1394 (1996).

[14] M. Horn-von Hoegen, F. K. LeGoues, M. Copel, M. C. Reuter, and R. M. Tromp, Phys. Rev. Lett. 67, 1130 (1991).

[15] G. Meyer, B. Voigtländer, and N.M. Amer, Surf. Sci. 274, L541 (1992).

[16] B. Voigtländer and A. Zinner, J. Vac. Sci. Technol. A 12, 1932 (1994).

[17] M. Horn-von Hoegen et al., Surf. Sci. 298, 29 (1993).

[18] M. Horn-von Hoegen and M. Henzler, Phys. Status Solidi A 146, 337 (1994).

[19] Th. Schmidt et al., Appl. Phys. Lett. 74, 1391 (1999).

[20] A. Janzen, I. Dumkow, and M. Horn-von Hoegen, Appl. Phys. Lett. 79, 2387 (2001).

[21] Th. Schmidt et al., Appl. Phys. Lett. 86, 111910 (2005).

[22] M. Horn-von Hoegen et al., Surf. Sci. 284, 53 (1993).

[23] Semiconductors - Basic Data, edited by O. Madelung (Springer, Berlin, 1996).

[24] M. Horn-von Hoegen, M. Copel, J.C. Tsang, M. C. Reuter, and R.M. Tromp, Phys. Rev. B 50, 10811 (1994).

[25] P. Zahl, P. Kury, and M. Horn-von Hoegen, Appl. Phys. A 69, 481 (1999).

[26] P. N. Keating, Phys. Rev. 145, 637 (1966).

[27] R. V. Hogg and A. T. Craig, Introduction to Mathematical Statistics (Macmillan, New York, 1978), 4th ed., see Sec. 3.3. 